\title{
Concentration and Solvency Effects on the Pair Interaction between Colloidal Particles in a Solution of Nonadsorbing Polymer
}

\author{
Remco Tuinier*,† and Gerard J. Fleer ${ }^{\ddagger}$ \\ Forschungszentrum Jülich, Institut für Festkörperforschung, 52425 Jülich, Germany, and \\ Laboratory for Physical Chemistry and Colloid Science, Wageningen University, \\ 6703 HB Wageningen, The Netherlands \\ Received July 14, 2004; Revised Manuscript Received August 31, 2004
}

\begin{abstract}
We present a simple analytical mean-field theory for the pair interaction between two colloidal particles, based upon a recent mean-field equation for the depletion thickness $\delta$ which depends on the chain length $N$, the bulk concentration $\varphi_{\mathrm{b}}$, and the solvency $\chi$. Only in the extremely dilute case is (in mean field) the interaction independent of $\chi$. At relevant concentrations a better solvency leads for two flat plates to a stronger attraction at contact $\left(\sim \varphi_{\mathrm{b}} / \delta\right)$ and a smaller range of attraction. This range is $2 \delta_{\mathrm{i}}$, where the "interaction distance" $\delta_{\mathrm{i}}$ is in semidilute solutions larger than $\delta$. In the dilute limit, both $\delta$ and $\delta_{\mathrm{i}}$ reduce to depletion thickness $\delta_{0}$ of ideal chains. The pair potential for flat plates can be described by a modified Asakura-Oosawa equation, in which $\delta_{\mathrm{i}}$ takes the place of the original $\delta_{0}$; this replacement accounts for the concentration and solvency dependence. For the interaction between two spheres of radius $a$ the contact potential is of order $a \varphi_{\mathrm{b}}$ and nearly insensitive to solvency; again, the range of attraction is smaller for better solvents. For two spheres we calculate the second virial coefficient as a function of concentration and solvent quality and its consequences for the stability of a colloidal dispersion at low colloid concentrations. For relatively short polymer chains the solvent quality hardly matters. For intermediate and large polymer-to-colloid size ratios, increasing the solvent quality leads to an increased miscibility. This implies that the increase in the osmotic pressure for polymers in a good solvent is overcompensated by a decrease of the depletion thickness, leading to weaker interactions.
\end{abstract}

\section{Introduction}

The free energy of interaction between two colloidal particles is the starting point for understanding colloidal stability. ${ }^{1}$ Even when colloidal dispersions are stable in pure solvent, the addition of nonadsorbing polymer may destabilize the dispersion when a certain polymer concentration is exceeded. This instability can be explained and quantified by the depletion interaction concept. Asakura and Oosawa ${ }^{2}$ were the first to calculate the depletion force between parallel plates due to ideal chains. Later, they also considered the pair interaction between two colloidal spheres; here the polymer chains were modeled as hard spheres (in the low-density limit) or as rods (to mimic stiff polymers). ${ }^{3}$ $\mathrm{Vrij}^{4}$ considered the interaction between two spheres in a sea of freely overlapping polymer spheres. Eisenriegler derived analytical expressions for the force between two spheres in a solution of ideal chains in the limit of large ${ }^{5}$ and small ${ }^{6}$ spheres. Using a superposition approximation, Tuinier et al. $^{7}$ calculated the pair interaction between two spherical colloids in a solution of ideal chains for arbitrary size ratios. Bolhuis et al. ${ }^{8}$ and Louis et al. ${ }^{9}$ computed the interaction between parallel plates and between colloidal spheres using a Gaussian core model for the nonadsorbing chains and performed computer simulations with long polymer chains in the excluded-volume limit. For an arbitrary size ratio, Fuchs and Schweizer introduced a two-component macromolecular integral theory approach, ${ }^{10}$ and Tuinier et al. ${ }^{11,12}$ used the adsorption method and included renormalization group results to predict pair interactions that agree well with computer simulation results. ${ }^{12}$

\footnotetext{
Institut für Festkörperforschung.

$\$$ Wageningen University.
}

In summary, there are several results for the ideal and excluded-volume limits, but there is much less insight into the polymer solvency effect on the interactions between colloidal particles. Scheutjens and Fleer ${ }^{13}$ performed numerical lattice calculations to calculate the depletion thickness at one plate and the interaction between two flat plates in the presence of nonadsorbing mean-field chains, using self-consistentfield (SCF) theory; in their model the solvent quality is included through the Flory-Huggins parameter $\chi$. Recently, we derived an analytical mean-field equation for the depletion thickness at a flat plate ${ }^{14}$ that agrees very well with these numerical SCF calculations. In the preceding paper ${ }^{15}$ we applied this equation to obtain simple analytical expressions for the excess amount and the grand potential for a single (flat or spherical) particle, and we found that these describe the exact results quite well. Here we extend this treatment to the interaction between two parallel plates and between two spherical particles. Where possible (i.e., for flat plates) we compare with numerical lattice results. The interaction potential is subsequently used to compute the osmotic second virial coefficient, which is a measure for the colloidal stability in dilute colloidal dispersions. In a future paper we intend to address many-body interactions in the phase behavior of colloidal spheres in a mean-field polymer solution and compute binodal curves.

In the present paper we will use several equations of the preceding paper, ${ }^{15}$ which we call part I. In most cases we do not repeat these equations; we refer to them by the equation number preceded by I.

\section{Basic Equations}

The pair potential $W(h)$ between two flat walls at a separation $h$ is given by $W(h)=2[\gamma(h)-\gamma(\infty)]$, where $\gamma(h)$ is the surface free energy per plate and the factor 
2 accounts for the two surfaces. In terms of the grand potential $\Omega$, which is the difference between $\gamma$ in the polymer solution and $\gamma_{0}$ in pure solvent, we may write

$$
W(h)=2[\Omega(h)-\Omega(\infty)]=-2 \Omega(\infty)\left[1-\frac{\Omega(h)}{\Omega(\infty)}\right]
$$

For $h=0$ we have $\Omega(0)=0$, so that the contact potential $W(0)$ is given by

$$
W(0)=-2 \Omega(\infty)
$$

The grand potential can be obtained from either the osmotic route or the adsorption method. For the osmotic route the generalization of eq I.3.2 to two plates is

$$
\Omega(h)=\int_{0}^{h / 2} \omega(z) \mathrm{d} z=\int_{0}^{h / 2}\left[\Pi_{\mathrm{b}}-\Pi(z)\right] \mathrm{d} z
$$

where the grand potential density profile $\omega(z)$ is coupled to the osmotic pressure profile $\Pi(z)$ in the slit; both depend on $h$ since $\varphi(z)=\varphi(z, h)$ is required as input.

In the adsorption method we use Gibbs' law:

$$
\mathrm{d} \Omega(h)=-\Gamma(h) \mathrm{d} \mu
$$

where $\Gamma(h)$ is the excess amount per plate, which is negative for depletion. When $\Gamma$ is expressed per segment, the chemical potential $\mu$ of the polymer is also per segment.

The deficit of polymer in the slit per unit area, $-2 \Gamma(h)$, equals $h$ times the concentration difference $\varphi_{\mathrm{b}}-\bar{\varphi}=\varphi_{\mathrm{b}}(1-K)$, where $\varphi_{\mathrm{b}}$ is the bulk concentration, $\bar{\varphi}$ the average concentration inside the slit, and $K \equiv \bar{\varphi} / \varphi_{\mathrm{b}}$ is the distribution coefficient, which is extensively used in size-exclusion chromatography (SEC). Therefore

$$
-\frac{\Gamma(h)}{\varphi_{\mathrm{b}}}=\frac{h}{2}(1-K)
$$

For wide slits where the depletion layers do not overlap, effectively a layer with thickness $\delta$, the single-plate depletion thickness, is void of polymer at each slit surface. Therefore, $\bar{\varphi}$ is lower than $\varphi_{\mathrm{b}}$ by a factor $(h-$ $2 \delta) / h=1-2 \delta / h$ :

$$
1-K=\frac{2 \delta}{h} \quad(\text { large } h)
$$

Substitution of eq 2.6 into eq 2.5 gives the excess amount $\Gamma(\infty)$ at a single plate, which was also used in part I (eq I.2.7):

$$
-\frac{\Gamma(\infty)}{\varphi_{\mathrm{b}}}=\delta
$$

All the above equations are completely general. To make progress, we have to find an expression for the depletion thickness $\delta$ and the distribution coefficient $K$. In this paper we wil use eq I.2.1 for $\delta=\delta\left(N, \varphi_{\mathrm{b}}, \chi\right)$ (see Figure 1 of part I for a graphical representation), and $K$ will be discussed below.

\section{Ideal Chains between Plates}

3.1. Asakura-Oosawa Equation. For ideal chains the osmotic pressure in eq 2.3 is given by the Van't Hoff law:

$$
\Pi=\frac{\varphi}{N} ; \quad \Pi_{\mathrm{b}}=\frac{\varphi_{\mathrm{b}}}{N}
$$

The integral over $\Pi_{\mathrm{b}}$ in eq 2.3 gives $\left(\varphi_{\mathrm{b}} / N\right) h / 2$, and the integral over $\Pi(z)$ leads to $(\bar{\varphi} / N) h / 2=K_{0}\left(\varphi_{\mathrm{b}} / N\right) h / 2$, where we use the index 0 to indicate the dilute limit of $K$. Therefore, the grand potential for ideal chains is given by

$$
\Omega(h)=\Pi_{\mathrm{b}} \frac{h}{2}\left[1-K_{0}\right]
$$

Equation 3.2 follows also from the adsorption method. From eqs 2.4 and 2.5 we have $\mathrm{d} \Omega(h)=(h / 2)\left(1-K_{0}\right) \varphi_{\mathrm{b}}$ $\mathrm{d} \mu$. In ideal solutions $\mu=N^{-1} \ln \varphi_{\mathrm{b}}$ (per segment), so $\varphi_{\mathrm{b}}$ $\mathrm{d} \mu=N^{-1} \mathrm{~d} \varphi_{\mathrm{b}}$. Then $\mathrm{d} \Omega / \mathrm{d} \varphi$ does not depend on $\varphi$, and integration of $\mathrm{d} \Omega$ between the limits $\varphi=0$ and $\varphi=\varphi_{\mathrm{b}}$ gives eq 3.2.

The distribution coefficient $K_{0}$ for Gaussian chains was derived by Casassa ${ }^{16}$ in the context of liquid chromatography of polymers. With the boundary condition of zero polymer concentration at the two walls, the result is

$$
\begin{aligned}
K_{0}=\frac{\bar{\varphi}}{\varphi_{\mathrm{b}}}=\frac{8}{\pi^{2}} \sum_{i=1,3, \ldots} \frac{1}{i^{2}} \mathrm{e}^{-\pi^{3}\left(i \delta_{0} / 2 h\right)^{2}}= \\
\begin{cases}\frac{8}{\pi^{2}} \mathrm{e}^{-\pi^{3}\left(\delta_{0} / 2 h\right)^{2}} & \frac{2 \delta_{0}}{h}>0.5 \\
1-\frac{2 \delta_{0}}{h} & \frac{2 \delta_{0}}{h}<0.5\end{cases}
\end{aligned}
$$

We will refer to this equation as the classical Casassa equation. As in the previous paper, $\delta_{0}=\sqrt{2 N / 3 \pi}=$ $(2 / \sqrt{\pi}) R$ (eq I.2.2) is the depletion thickness of ideal chains at a single surface. Again we express all lengths in units $l$, the monomer length. The narrow-slit limit $\left(2 \delta_{0} / h>0.5\right)$ for $K_{0}$ is just the first term of the summation in the full expression; it gives $K_{0}=0.499$ for $2 \delta_{0} / h=0.5$. The wide-slit limit $\left(2 \delta_{0} / h<0.5\right)$ describes the situation that the two depletion layers do not overlap; it gives $K_{0}=0.500$ for $2 \delta_{0} / h=0.5$. Hence, the two limiting forms of eq 3.3 cross over very smoothly, and the second version of eq 3.3 is nearly exact. An important feature of eq 3.3 is that $K_{0}$ depends only on the ratio $h / \delta_{0}$.

Substituting $1-K_{0}=2 \delta_{0} / h$ into eq 3.2 gives the grand potential for a single surface

$$
\Omega(\infty)=\Pi_{b} \delta_{0}
$$

which is identical to the dilute limit of eq I.3.10.

Combination of eq 2.1 with eqs 3.2 and 3.4 gives the exact result for the pair potential of ideal chains:

$$
\begin{aligned}
& W(h)=-2 \Omega(\infty)\left[1-\left(1-K_{0}\right) \frac{h}{2 \delta_{0}}\right]= \\
&-\Pi_{\mathrm{b}}\left[2 \delta_{0}-h\left(1-K_{0}\right)\right]
\end{aligned}
$$

where $W(h)$ is in units $k T / l^{2}$. This equation was first derived (in a different way) by Asakura and Oosawa. ${ }^{2}$ We will refer to it as the classical $\mathrm{AO}$ equation. It contains two parameters: $\delta_{0}$, defining the range of the interaction, and $\Pi_{\mathrm{b}}$ or $\Omega(\infty)=\Pi_{\mathrm{b}} \delta_{0}$, determining the contact potential $W(0)$. We note that for wide slits $\left(2 \delta_{0} / h\right.$ 


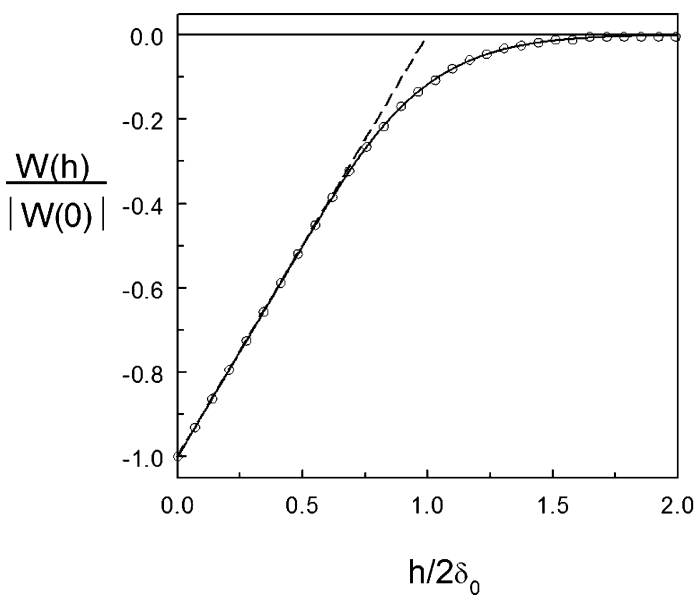

Figure 1. Pair potential $W(h)$ between two parallel plates immersed in a solution of ideal chains as a function of the plate separation $h$, according to eq 3.5 (solid curve). The pair potential was scaled by the contact potential $W(0)$ and the separation by the interaction range $2 \delta_{0}$. The data points are SCF lattice results for $\varphi_{\mathrm{b}}=10^{-3}, N=10^{3}$, and $\chi=0.5$. The dashed line is the linear dependence of eq 3.7.

$<0.5)$ we have $h\left(1-K_{0}\right)=2 \delta_{0}$ so that $W(h)=0$, as expected for nonoverlapping depletion layers.

In Figure 1 we compare eq 3.5 (solid curve) to numerical self-consistent-field (SCF) lattice results. ${ }^{17}$ We normalized $W(h)$ by $|W(0)|=2 \Omega(\infty)=2 \varphi_{\mathrm{b}} \delta_{0} / N$ and the plate separation $h$ by $2 \delta_{0}$. The SCF data (symbols) were computed for theta conditions $(\chi=0.5), N=1000$, and $\varphi_{\mathrm{b}}=10^{-3}$. To implement the boundary condition $\varphi(0)=0$ also in the lattice model, the adsorption energy parameter $\chi_{\mathrm{s}}$ was chosen as $\chi_{\mathrm{s}}=-(1+\chi) / 6$, as discussed previously. ${ }^{14,15}$

It is clear from Figure 1 that there is quantitative agreement between the continuum and lattice models. In the normalized presentation of this figure, the lattice results for $\chi=0.5$ are insensitive to the bulk concentration in the range $\varphi_{\mathrm{b}}<10^{-3}$, and for $\varphi_{\mathrm{b}}<10^{-4}$ there is no perceptible solvency effect of the pair interaction in the range $0<\chi<0.5$.

An alternative way of writing eq 3.5 is to replace $2 \delta_{0}$ by $-2 \Gamma(\infty) / \varphi_{\mathrm{b}}($ eq 2.7$)$ and $-h\left(1-K_{0}\right)$ by $2 \Gamma(h) / \varphi_{\mathrm{b}}$ (eq 2.5):

$$
W(h)=\frac{2 \Pi_{\mathrm{b}}}{\varphi_{\mathrm{b}}}[\Gamma(\infty)-\Gamma(h)]=\frac{2}{N}[\Gamma(\infty)-\Gamma(h)]
$$

According to eq 3.5 or 3.6 the contact potential equals $W(0)=-2 \Pi_{b} \delta_{0}$. We will see later (eq 4.8) that in the general case a similar result applies with for $\Pi_{b}$ the full mean-field expression. However, the length $\delta_{0}$ has to be replaced by an interaction distance $\delta_{\mathrm{i}}$ which is of the order of (but in semidilute solutions larger than) the single-plate depletion thickness $\delta$ as defined in eq I.2.1.

3.2. Step-Function Approach. The dashed line in Figure 1 shows that a reasonable first-order approximation for $W(h)$ at small $h$ is obtained from a step-function approach $\left(\varphi(z)=0\right.$ and $\bar{\varphi}=0$ inside the slit, $\varphi(z)=\varphi_{\mathrm{b}}$ outside). Then $K=\bar{\varphi} / \varphi_{\mathrm{b}}=0$. With this step function we get for ideal chains from eq 3.5

$$
\begin{aligned}
& W(h)=-2 \Omega(\infty)\left[1-\frac{h}{2 \delta_{0}}\right]= \\
&-\Pi_{\mathrm{b}}\left(2 \delta_{0}-h\right) \quad h<2 \delta_{0}
\end{aligned}
$$

Hence, the force $-\mathrm{d} W / \mathrm{d} h$ (per unit area) equals minus the osmotic pressure. We can write eq 3.7 in another way by introducing the overlap volume $V_{\text {ov }}$ of the two depletion layers (both considered to be step functions at a distance $\delta_{0}$ from the wall):

$$
V_{\mathrm{ov}}=A\left(2 \delta_{0}-h\right) ; \quad W(h)=-\Pi_{\mathrm{b}} \frac{V_{\mathrm{ov}}}{A} \quad h<2 \delta_{0}
$$

where $A$ is the area per plate. In section 4.3 we will see that eq 3.8 also applies to mean-field chains at finite concentrations, provided a more general expression for $\Pi_{\mathrm{b}}$ is used, and a different range $2 \delta_{\mathrm{i}}$ (where $\delta_{\mathrm{i}}$ is the "interaction distance") is taken to calculate $V_{\text {ov }}$. Equation 3.8 can also be used for the interaction between two spheres with, obviously, a different expression for $V_{\text {ov }}$.

In this step-function approach $\Gamma(h)-\Gamma(\infty)$ equals $\varphi_{\mathrm{b}}\left(\delta_{0}-h / 2\right)$ according to eqs 2.5 (with $1-K_{0}=1$ ) and 2.7. In combination with eq $3.8 \mathrm{a}$

$$
\Gamma(h)-\Gamma(\infty)=\frac{\varphi_{\mathrm{b}}}{2} \frac{V_{\mathrm{ov}}}{A}
$$

Also this equation is valid for spheres, using an appropriate expression for $V_{\text {ov }}$.

\section{Mean-Field Chains between Plates}

4.1. Adsorption Method. According to eq 2.2, the contact potential $(h=0)$ is generally given by $W(0)=$ $-2 \Omega(\infty)$. In part I (eq I.3.10) we found

$$
\Omega(\infty)=\frac{2 f}{9} \frac{\varphi_{\mathrm{b}}}{\delta} ; f=1-\left(1-\frac{3}{\pi}\right)\left(\frac{\delta}{\delta_{0}}\right)^{2}
$$

where the depletion thickness $\delta$ is defined in eq I.2.1. The factor $f$ in eq 4.1 varies from $3 / \pi=0.955$ in dilute solutions to 1 in concentrated systems.

For nonzero $h$ we combine eqs 2.1 and 2.4:

$$
\mathrm{d} W(h)=2[\Gamma(\infty)-\Gamma(h)] \mathrm{d} \mu
$$

where $\Gamma(h)$ in its general form has to be found from integration over the concentration profile in the slit. Using $\mathrm{d} \mu=(2 / 3 \pi) \delta_{\mathrm{c}}{ }^{2} \mathrm{~d} \varphi / \varphi$ as derived in eq I.3.7, with the compressibility thickness $\delta_{c}$ defined in eq I.3.6, we find the generalization of eq I.3.8 to two plates

$$
W(h)=\frac{4}{3 \pi} \int_{0}^{\varphi_{b}}\left(\frac{\Gamma(\infty)-\Gamma(h)}{\varphi} \frac{1}{\delta_{c}^{2}}\right) \mathrm{d} \varphi
$$

where $\Gamma(h)$ and $\delta_{\mathrm{c}}$ depend on the buildup concentration $\varphi$ in the system. Equation 4.3 as such is general, but in order to use it we need approximations since we have no general expression for the concentration profile in the slit. We note that eq 4.3 applies to arbitrary geometry. In the following section we consider only the planar case; in section 5 we apply eq 4.3 to spheres.

4.2. Step-Function Approach. When we adopt the step function approach, we may apply eq 2.5 with $1-$ $K=1$ and eq 2.7: $[\Gamma(\infty)-\Gamma(h)] / \varphi=h / 2-\delta$ for $h<2 \delta$. Using the Heaviside step function $H(x)$, which is zero for negative $x$ and unity for positive $x$, we may write

$$
\frac{\Gamma(\infty)-\Gamma(h)}{\varphi}=-\frac{2 \delta-h}{2} H(2 \delta-h)
$$




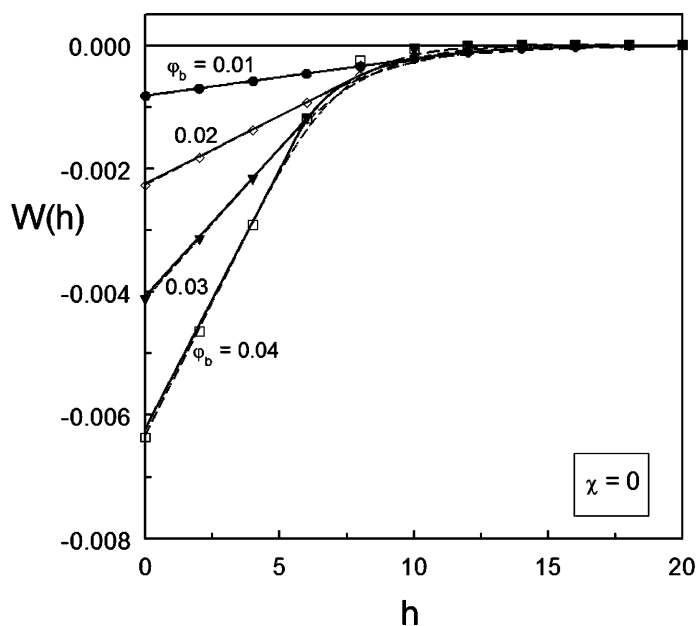

Figure 2. Pair potential between two parallel plates immersed in a solution of mean-field chains as a function of the plate separation $h$ according to eq 4.5 (solid curves) and eq 4.12 (dashed), for $N=10^{3}, \chi=0$, and four polymer concentrations as indicated in the plot. The data points are numerical SCF lattice results.

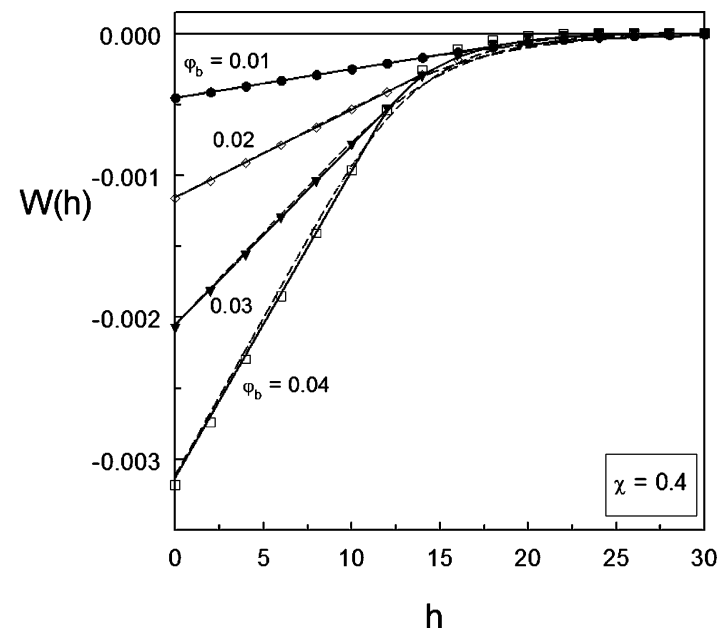

Figure 3. As Figure 2 but for $\chi=0.4$.

where $\delta=\delta(\varphi)$ decreases with increasing $\varphi$. Substitution into eq 4.3 gives

$$
W(h)=-\frac{2}{3 \pi} \int_{0}^{\varphi_{\mathrm{b}}} \frac{2 \delta-h}{\delta_{\mathrm{c}}^{2}} H(2 \delta-h) \mathrm{d} \varphi
$$

This equation requires a numerical integration. For $h$ $=0$, the contact potential $W(0)=-2 \Omega(\infty)$ with eq I.3.9 for $\Omega(\infty)$ is recovered, which may be transformed into eq 4.1 using Appendix 2 of paper I.

We note that at given $\varphi\left(0<\varphi<\varphi_{\mathrm{b}}\right)$ the integrand is a linear function of $h$ in the interval $0<h<2 \delta$ (and zero for $h>2 \delta$ ). However, the integral itself is not linear in $h$ since the value of $\delta$ (i.e., the width of the integration interval) depends on $\varphi$. For small $\varphi$ there are contributions over the range $0<h<2 \delta_{0}$; for higher $\varphi$ the interval decreases as $\delta$ becomes smaller. The result is that, even though $\mathrm{d} W(h) \sim(2 \delta-h) \mathrm{d} \varphi$ at given $\varphi$ is assumed to be linear in $h$ for $0<h<2 \delta$, the integrated form $W(h)$ is not linear but has a "tail" in the region $2 \delta$ $<h<2 \delta_{0}$.

Figures 2-4 compare the results of eq 4.5 (solid curves) for $N=1000$, four concentrations in the range $0.01-0.04$, and three solvencies $(\chi=0$ in Figure $2, \chi=$

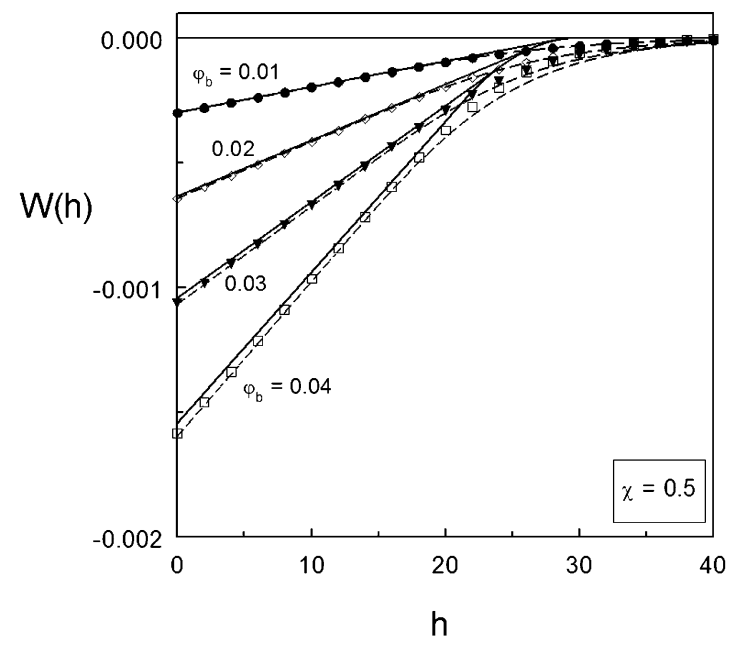

Figure 4. As Figure 2 but for $\chi=0.5$.

0.4 in Figure 3 , and $\chi=0.5$ in Figure 4 ) with SCF lattice results (calculated with $\chi_{\mathrm{s}}=-(1+\chi) / 6$ ). The agreement is excellent for strong overlap (where the assumption 1 $-K=0$ is justified) and quite satisfying for weak overlap: even the tail end of the pair potential is captured reasonably well, at least for good solvents. Only for $\chi=0.5$ does the analytical result underestimate $W(h)$ at the start of the interaction. This is due to the fact that the step-function approach works less well in a $\Theta$ solvent, in which the chains can easier interpenetrate than in a good solvent.

The dashed curves in Figures 2-4 were calculated with the modified $\mathrm{AO}$ equation, to be discussed in section 4.4.

It is clear from these figures that there is a strong solvency effect on both the contact potential $W(0)$ and the range of interaction. Both effects are easily understood and follow from the $\chi$ dependence of the depletion thickness $\delta$ (Figure 1 of part I). According to eq $4.1 \mathrm{~W}(0)$ is proportional to $\varphi_{\mathrm{b}} / \delta$ and, hence, higher in good solvents. On the other hand, the range is of order $2 \delta$ and thus smaller for better solvency. In the next section, we consider the interaction range in more detail.

4.3. Range of Interaction. For ideal chains we found $W(h)=-\Pi_{\mathrm{b}}\left(2 \delta_{0}-h\right)\left(\right.$ eq 3.7) for small $h$, where $2 \delta_{0}=$ $(4 / \sqrt{\pi}) R$ is the range of the interaction and $\Pi_{\mathrm{b}}$ the (ideal) osmotic pressure. From the numerical SCF results (Figures 2-4) we see for mean-field chains again a linear dependence for small $h$, which thus also can be described by two parameters.

The first is the range, which is smaller than $2 \delta_{0}$. We expect this range to be of order $2 \delta$ because $\delta$ is the appropriate length scale for single surfaces and wide slits. However, the length scale for strongly interacting depletion layers (nearly empty slits) is not necessarily the same. Let us call the range for narrow slits $2 \delta_{\mathrm{i}}$, where $\delta_{\mathrm{i}}$ is the "interaction distance". Below (eq 4.9) we will find an expression for it.

The second parameter is the osmotic pressure, for which we need a more general equation. From the Flory-Huggins expression (eq I.3.3)

$$
\begin{aligned}
& \Pi_{\mathrm{b}}=-\ln \left(1-\varphi_{\mathrm{b}}\right)-\varphi_{\mathrm{b}}\left(1-\frac{1}{N}\right)-\chi \varphi_{\mathrm{b}}{ }^{2}= \\
& \frac{\varphi_{\mathrm{b}}}{N}+\frac{1}{2} v \varphi_{\mathrm{b}}{ }^{2}+\frac{1}{3} \varphi_{\mathrm{b}}{ }^{3}+\ldots
\end{aligned}
$$


We can now generalize eq 3.7 to

$$
W(h)=-2 \Omega(\infty)\left[1-\frac{h}{2 \delta_{\mathrm{i}}}\right]=-\Pi_{\mathrm{b}}\left[2 \delta_{\mathrm{i}}-h\right]
$$

where $\Omega(\infty)$ is given by eq 4.1 . This initial linear part of $W(h)$ extrapolates to $W(h)=0$ at $h=2 \delta_{\text {i }}$.

The contact potential is now given by

$$
W(0)=-2 \Omega(\infty)=-2 \Pi_{\mathrm{b}} \delta_{\mathrm{i}}
$$

This is the same result as found from the "overlap Ansatz" (eq 3.8) with $V_{\text {ov }}(0)=2 A \delta_{\mathrm{i}}$. We note, however, that there is a small (but in semidilute solutions significant) difference with the simplistic approach of calculating $V_{\text {ov }}$ from two overlapping depletion layers with a thickness corresponding to the single-layer depletion thickness $\delta$. We will see below that $\delta_{\mathrm{i}}$ is larger than $\delta$ by roughly a factor $4 / 3$ and $3 / 2$ (see eq 4.10 ) in semidilute solutions in good and $\Theta$ solvents, respectively.

According to eq $4.8 \delta_{\mathrm{i}}$ is defined as

$$
\delta_{\mathrm{i}}=\frac{\Omega(\infty)}{\Pi_{\mathrm{b}}}=\frac{\Omega(\infty) / \delta}{\Pi_{\mathrm{b}}} \delta
$$

This definition of $\delta_{\mathrm{i}}$ is identical to that of Joanny et al., ${ }^{18}$ also used by Louis et al. ${ }^{9}$ (their eqs 4 plus 5 ). In the dilute limit $\left(\Omega(\infty)=\Pi_{\mathrm{b}} \delta_{0}\right.$, see eq 3.4) $\delta_{\mathrm{i}}$ equals $\delta_{0}$.

The ratio $\Omega(\infty) / \delta$ equals $\varphi_{\mathrm{b}} / N+(2 / 3) v \varphi_{\mathrm{b}}{ }^{2}+(23 / 45) \varphi_{\mathrm{b}}{ }^{3}$ (eq A2 of part I), and for $\Pi_{\mathrm{b}}$ we have $\Pi_{\mathrm{b}}=\varphi_{\mathrm{b}} / N+(1 /$ 2) $v \varphi_{\mathrm{b}}{ }^{2}+(1 / 3) \varphi_{\mathrm{b}}{ }^{3}$ (eq 4.6). The ratio $\delta_{\mathrm{i}} / \delta$ is thus of order unity, but for finite concentrations it is higher:

$$
\frac{\delta_{\mathrm{i}}}{\delta} \approx \frac{1 / N+(2 / 3) v \varphi_{\mathrm{b}}+(1 / 2) \varphi_{\mathrm{b}}{ }^{2}}{1 / N+(1 / 2) v \varphi_{\mathrm{b}}+(1 / 3) \varphi_{\mathrm{b}}{ }^{2}}
$$

In the dilute limit $\delta_{\mathrm{i}} / \delta=1$, at semidilute concentrations in a good solvent $\delta_{\mathrm{i}} / \delta \approx 4 / 3$ for long chains, and under semidilute theta conditions this ratio is about $3 /{ }_{2}$ for $N$ $\rightarrow \infty$.

A comparison of $\delta_{\mathrm{i}}$ and $\delta$ as a function of concentration and solvency for $N=600$ is given in Figure 5 . The solid curves represent the interaction distance $\delta_{\mathrm{i}}$ as calculated from eq 4.9 with eq 4.1 for $\Omega(\infty)$ and eq 4.6 for $\Pi_{b}$. The symbols in Figure 5 are SCF lattice results for $\delta_{i}$, obtained with the numerically exact values for $\Omega(\infty)$. It is clear that there is excellent agreement between these two sets of data. In Figure 5 we plotted for comparison also the depletion thickness $\delta$ according to eq I.2.1 (dashed curves). As anticipated from eq 4.10, $\delta_{\mathrm{i}}$ is larger than $\delta$ for polymer concentrations where both lengths are smaller than $\delta_{0}$.

From Figures 2-4 we saw that the adsorption method (eq 4.5), which takes into account the buildup of the system by gradually increasing the polymer concentration (at the same time assuming that at any concentration a step function at distance $\delta$ may be used), is in excellent agreement with the exact SCF results. Apparently, the integration corresponding to this buildup gives a better description than the simplistic assumption $V_{\text {ov }}(0)=2 A \delta$, with $\delta$ only determined by the final concentration $\varphi_{\mathrm{b}}$. Effectively, this integration defines an effective interaction range $\delta_{\mathrm{i}}$ which is some weighted average of the highest value $\delta_{0}$ in the dilute limit and the lowest value $\delta$ corresponding to $\varphi_{\mathrm{b}}$; that is why the ratio $\delta_{\mathrm{i}} / \delta$ is above unity.

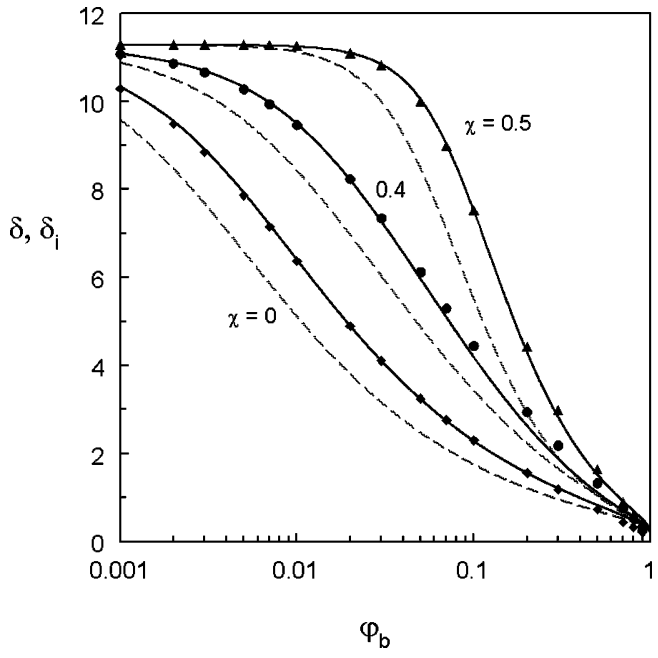

Figure 5. Interaction distance $\delta_{\mathrm{i}}$ (solid curves and data points) and depletion thickness $\delta$ (dashed curves) as a function of the polymer concentration, for three solvencies as indicated, and $N=600\left(R=10, \delta_{0}=20 / \sqrt{\pi}=11.28\right)$. The solid curves were computed from eq 4.9 , with eq 4.1 for $\Omega(\infty)$ and eq 4.6 for the osmotic pressure $\Pi_{b}$. The symbols are SCF results calculated from the numerically exact $\Omega(\infty)$. The dashed curves for $\delta$ follow eq I.1.2.

4.4. Modified Asakura-Oosawa Equation. Knowing the proper length scale $\delta_{\mathrm{i}}$ for narrow slits, we can now also generalize the classical AO equation (eq 3.5) outside the linear region $(1-K=1)$ as described by eq 4.7. To that end we replace $K_{0}\left(\delta_{0}\right)$ in eq 3.5 by $K\left(\delta_{\mathrm{i}}\right)$. The latter quantity is found by introducing a modified Casassa equation, replacing $\delta_{0}$ in the classical Casassa equation (eq 3.3) by $\delta_{\mathrm{i}}$ :

$$
K= \begin{cases}\frac{8}{\pi^{2}} \mathrm{e}^{-\pi^{3}\left(\delta_{\mathrm{i}} / 2 h\right)^{2}} & \frac{2 \delta_{\mathrm{i}}}{h}>0.5 \\ 1-\frac{2 \delta_{\mathrm{i}}}{h} & \frac{2 \delta_{\mathrm{i}}}{h}<0.5\end{cases}
$$

Inserting this into eq 3.5 , we obtain the modified $\mathrm{AO}$ equation:

$$
\begin{aligned}
& W(h)=-2 \Omega(\infty)\left[1-(1-K) \frac{h}{2 \delta_{\mathrm{i}}}\right]= \\
&-\Pi_{\mathrm{b}}\left[2 \delta_{\mathrm{i}}-h(1-K)\right]
\end{aligned}
$$

The dashed curves in Figures $2-4$ give $W(h)$ according to the simple explicit eq 4.12. The agreement with the numerical SCF data is of the same level as the numerical integration of eq 4.5 (and in a $\Theta$ solvent is even better). So, when we would be interested only in flat geometry, we could use just eq 4.12 and forget about the more complicated adsorption method (eq 4.5). However, for the interaction between spheres (section 5) we have no modified $\mathrm{AO}$ equation, and the adsorption method still works.

According to eq 4.12, the essential features of the pair potential between planar surfaces are just two parameters: $W(0)=-2 \Omega(\infty)$ and $\delta_{\mathrm{i}}$. The ratio $W(h) / W(0)$ is only a function of $h / \delta_{\mathrm{i}}$. Hence, when $W(h)$ is normalized by $W(0)$ and $h$ by $\delta_{i}$, we may expect one master curve for all the data of Figures 2-4. We illustrate this in Figure 6 , where the symbols represent numerical SCF data: for $\Omega(\infty)$ we took the numerical value, and $\delta_{\mathrm{i}}$ was obtained as $\Omega(\infty) / \Pi_{\mathrm{b}}$. Except for some small deviations 


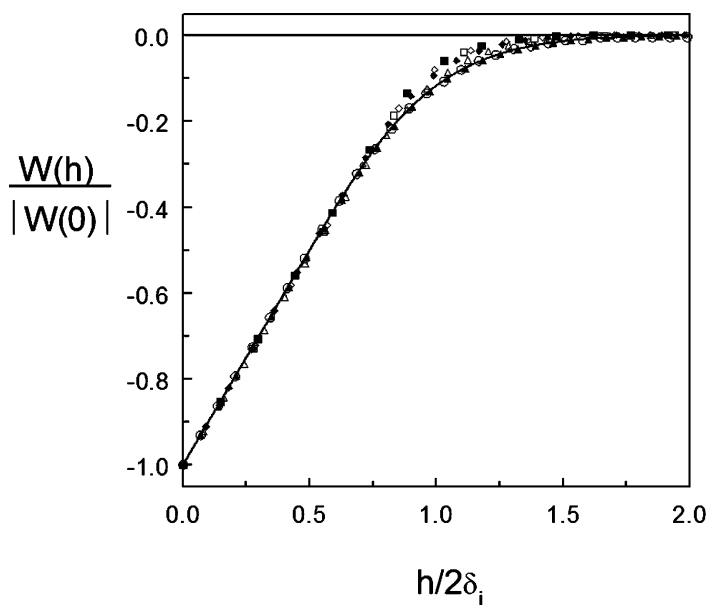

Figure 6. Master curve for the pair potential between two plates for all the data points of Figures $2-4$ for $\varphi_{\mathrm{b}}=0.01$ and 0.04 . The pair potential $W(h)$ and the scaling parameters $W(0)$ $=-2 \Omega(\infty)$ and $\delta_{\mathrm{i}}$ were computed from numerically exact SCF lattice data for the conditions of Figures 2-4: $\chi=0$, squares; $\chi=0.4$, diamonds; $\chi=0.5$, triangles. Filled symbols represent $\varphi_{\mathrm{b}}=0.01$, and open symbols correspond to $\varphi_{\mathrm{b}}=0.04$. The solid curve is the modified $\mathrm{AO}$ equation (eq 4.12).

around $h=2 \delta_{\mathrm{i}}$, we do indeed find a single master curve, which strongly resembles Figure 1.

The solid curve in Figure 6 is drawn according to eq 4.12. Apart from the (small) deviations around $h / 2 \delta_{\mathrm{i}}=$ 1 , the agreement with the numerical SCF results is excellent. Hence, our simple modified $\mathrm{AO}$ equation gives a very good description of the pair potential between two plates.

It is possible to analyze the nonuniversal effects around $h / 2 \delta_{i}=1$ in more detail. We will not do that here but we refer to Fleer and Skvortsov. ${ }^{19}$ We just mention that two length scales play a role in this transition region between wide slits (length scale $\delta$ ) where the pair potential is zero and narrow slits (length scale $\delta_{\mathrm{i}}$ ) where the pair potential is linear in $h$. The implication is that the use of eq 4.11 to arrive at the modified AO equation is a reasonable approximation for the pair potential, where the physics are dominated by the narrow-slit regime because $W(h)=0$ for wide slits $\left(h>4 \delta_{\mathrm{i}}\right)$, but is inaccurate as to the distribution coefficient $K$ in wide pores (which is the important regime in SEC). For the distribution coefficient in SEC a modified Casassa equation as eq 4.11 but with $\delta_{\mathrm{i}}$ replaced be the wide-slit length scale $\delta$ is more accurate. $^{19}$

\section{Interaction between Two Spheres}

5.1. Pair Potential. For the interaction between two spheres, the situation is more complicated. Even for ideal chains there is no full analytical solution which is the counterpart of the classical AO equation, eq 3.5 (see, however, eq 5.8, which gives an exact result for the contact potential of large spheres). In this case there are also hardly any lattice results available: some computations using a 2D cylindrical lattice have been done, ${ }^{20}$ but they are limited to very small spheres and short polymer chains. Hence, we cannot check the assumptions made in deriving analytical approximations against exact SCF results.

We treat this problem again on the level of the stepfunction approach, using the spherical analogue of eq 3.9 , in which the overlap volume $V_{\text {ov }}$ occurs. Whereas for planar geometry $\Gamma(h)$ and $W(h)$ were expressed per

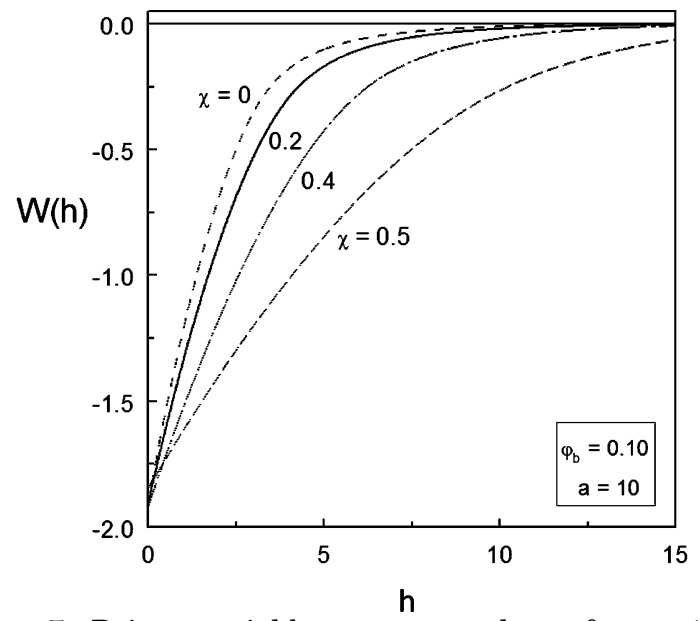

Figure 7. Pair potential between two spheres for $a=10, \varphi_{\mathrm{b}}$ $=0.10, N=10^{3}$, and four solvencies $(\chi=0,0.2,0.4$, and 0.5$)$ according to eq 5.4 .

unit area, with $\Gamma / \varphi_{\mathrm{b}}$ in units $l$ and $W$ in units $k T / l^{2}$, for spheres we consider the total depleted amount $\left(\Gamma / \varphi_{\mathrm{b}}\right.$ in units $l^{3}$ ) and pair potential $W_{\mathrm{s}}$ (in units $k T$ ). Note that $\Gamma$ as used here for spheres equals $\Gamma_{\mathrm{s}}$ (per unit area, used in part I) times $4 \pi a^{2}$. Equation 3.9 is now modified to

$$
2[\Gamma(h)-\Gamma(\infty)]=\varphi_{\mathrm{b}} V_{\mathrm{ov}}
$$

where $\Gamma(h)$ is the (total) depleted amount per sphere. We assume that we may calculate $V_{\text {ov }}$ as the overlap volume of two spherical depletion shells with inner radius $a$ (the particle radius) and outer radius $a+\delta_{\mathrm{s}}$, where $\delta_{\mathrm{s}}$ is the depletion thickness around a sphere, calculated according to eq I.4.6:

$$
\left(1+\frac{\delta_{\mathrm{s}}}{a}\right)^{3}=1+3 \frac{\delta}{a}+\frac{\pi^{2}}{4} \frac{\delta_{\mathrm{c}}^{2}}{a^{2}}
$$

where $\delta$ is given by eq I.2.1 and $\delta_{\mathrm{c}}$ is defined in eq I.3.6.

For large $a$ (colloid limit) $\delta_{\mathrm{s}}$ approximately equals the "flat" depletion thickness $\delta$, and for smaller particles it is smaller; see also Figure 7 in ref 14 (where eq 5.2 was used with $\delta$ also in the curvature term).

Clearly, $V_{\text {ov }}$ depends on the distance $h$ between the two sphere surfaces. From elementary geometry we have

$$
V_{\mathrm{ov}}=\frac{\pi}{12}\left(2 \delta_{\mathrm{s}}-h\right)^{2}\left(6 a+4 \delta_{\mathrm{s}}+h\right) H\left(2 \delta_{\mathrm{s}}-h\right)
$$

where the Heaviside function ensures that $V_{\text {ov }}$ is zero when the two depletion layers do not overlap.

We can now substitute eq 5.1 into eq 4.3 to find

$$
W_{\mathrm{s}}(h)=-\frac{2}{3 \pi} \int_{0}^{\varphi_{\mathrm{b}}} \frac{V_{\mathrm{ov}}}{\delta_{\mathrm{c}}{ }^{2}} \mathrm{~d} \varphi
$$

where both $\delta_{\mathrm{c}}$ and $V_{\mathrm{ov}}$ (containing $\delta_{\mathrm{s}}$, which is a function of $\delta$ and $\delta_{\mathrm{c}}$ ) depend on the integration variable (buildup concentration) $\varphi$. Equation 5.4 has to be evaluated numerically.

Some results of eq 5.4 for $W_{\mathrm{s}}(h)$ are given in Figures 7 and 8. Figure 7 shows $W_{\mathrm{s}}(h)$ for $N=1000, a=10, \varphi_{\mathrm{b}}$ $=0.1$, and four solvencies in the range $\chi=0-0.5$. It turns out that the contact potential $W_{\mathrm{s}}(0)$ does hardly depend on solvency, whereas the interaction range increases when the solvent becomes poorer. The latter effect is easily understood: the most important factor 


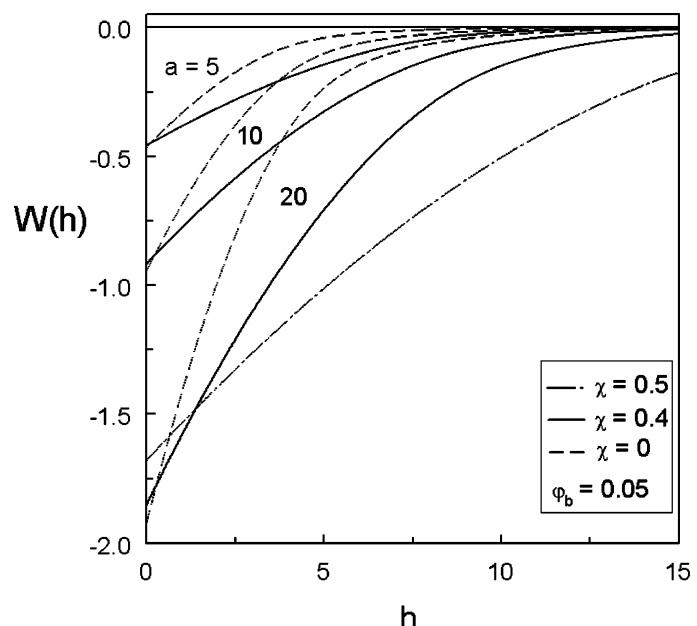

Figure 8. Pair potential (eq 5.4) between two spheres for $a$ $=5,10$, and $20, \varphi_{\mathrm{b}}=0.05, N=10^{3}$, and two solvencies: $\chi=$ 0 (dashed curves) and $\chi=0.4$ (solid curves). The dot-dash curve gives the pair potential for $a=20$ and $\chi=0.5$.

in the decay of $W_{\mathrm{s}}(h)$ is the factor $\left(2 \delta_{\mathrm{s}}-h\right)^{2}$ in $V_{\mathrm{ov}}$, which shows that the range is of order $2 \delta_{\mathrm{s}}$; like $\delta, \delta_{\mathrm{s}}$ becomes smaller in better solvents. We will demonstrate below that the range is higher than $2 \delta_{\mathrm{s}}$ because during the integration $\delta_{\mathrm{s}}$ decreases from its highest value $\delta_{\mathrm{s}}(\varphi=$ $0)$ to its lowest value $\delta_{\mathrm{s}}\left(\varphi=\varphi_{\mathrm{b}}\right)$. We will also see why $W_{\mathrm{s}}(0)$ is about the same for all solvencies.

In Figure 8 we present, for a lower concentration $\varphi_{\mathrm{b}}$ $=0.05$, the effect of the particle radius $(a=5,10,20)$ for $\chi=0$ (dashed curves) and $\chi=0.4$ (solid); we also show one curve $(a=20)$ for $\chi=0.5$ (dash-dot). Again we see the approximate independence of $W_{\mathrm{s}}(0)$ on solvency. Comparison of the curves for $a=10$ with those in Figure 7 shows that $W_{\mathrm{s}}(0)$ is roughly proportional to the bulk concentration $\varphi_{\mathrm{b}}$. Moreover, from Figure 8 it follows that $W_{\mathrm{s}}(0)$ and $W_{\mathrm{s}}(h)$ are nearly proportional to the particle size. We analyze these effects below.

5.2. Contact Potential. The contact potential $W_{\mathrm{s}}(0)$ is found by inserting $V_{\mathrm{ov}}=(2 \pi / 3) \delta_{\mathrm{s}}{ }^{2}\left(3 a+2 \delta_{\mathrm{s}}\right)$ into eq 5.4:

$$
W_{\mathrm{s}}(0)=-\frac{4}{3} a \int_{0}^{\varphi_{\mathrm{b}}} \frac{\delta_{\mathrm{s}}{ }^{2}}{\delta_{\mathrm{c}}{ }^{2}}\left(1+\frac{2 \delta_{\mathrm{s}}}{3 a}\right) \mathrm{d} \varphi
$$

The integrand of eq 5.5 depends only weakly on concentration or solvency. This is most easily seen for relatively large $a\left(\delta_{\mathrm{s}} \approx \delta\right)$, where the integrand reduces to $\left(\delta / \delta_{\mathrm{c}}\right)^{2}$. Let us first consider the dilute limit $\left(\delta_{\mathrm{c}}=\delta=\right.$ $\left.\delta_{0}\right)$. Then eq 5.5 simplifies to

$$
W_{\mathrm{s}}(0)=-\frac{4}{3} a \varphi_{\mathrm{b}}
$$

This equation can also be derived from the flat-plate result for $W(h)$ for low $h$ (eq 3.7) in the dilute limit, $W(h)$ $=W(0)\left(1-h / 2 \delta_{0}\right)$, using the Deryagin approximation ${ }^{21}$ which in this case reads

$$
W_{\mathrm{s}}(h)=\pi a \int_{h}^{2 \delta_{0}} W\left(h^{\prime}\right) \mathrm{d} h^{\prime}
$$

Applying this equation for $h=0$ gives immediately eq 5.6, showing the internal consistency of our procedure.

Equation 5.6 is quite close to an exact result derived by Eisenriegler. ${ }^{5} \mathrm{He}$ inserted the classical $\mathrm{AO}$ equation (eq 3.5) into eq 5.7 (with integration limits 0 and $\infty$ ) and found for the limit of low polymer concentration and large radius:

$$
W_{\mathrm{s}}(0)=-4 \pi \ln (2) \frac{R^{2}}{N} a \varphi_{\mathrm{b}}
$$

With $R^{2} / N=1 / 6$, the numerical prefactor in eq 5.8 is $(4 / 3)(\pi / 2) \ln 2$, which is only a factor $(\pi / 2) \ln 2=1.089$ different from eq 5.6. This good agreement leads us to believe that our treatment is also reasonable for nonideal chains.

For finite concentrations eq 5.6 no longer holds, but the changes are not dramatic because $\delta_{\mathrm{s}} / \delta_{\mathrm{c}}$ is of order unity. For large particles the integrand of eq 5.5 starts at 1 for $\varphi=0$ and passes through a weak maximum (for $N=1000$ this maximum is about 1.5 at $\varphi \approx 0.03$ for $\chi=0$ and around 1.8 at $\varphi \approx 0.14$ for $\chi=0.5$ ), after which it decreases very slowly. For smaller particles the effect is roughly the same, with a starting value which is only slightly higher (e.g., 1.40 for $a=1$ and $N=1000$ ) and a maximum of about the same height which is shifted to lower $\varphi$. All in all, it is not too bad an approximation to take the integrand out of the integral (which boils down to assigning the value of $\delta_{\mathrm{s}}$ and $\delta_{\mathrm{c}}$ corresponding to $\varphi_{\mathrm{b}}$ to the entire integration interval). Then we get

$$
W_{\mathrm{s}}(0) \approx-\frac{4}{3} a \varphi_{\mathrm{b}} \frac{\delta_{\mathrm{s}}^{2}}{\delta_{\mathrm{c}}^{2}}\left(1+\frac{2 \delta_{\mathrm{s}}}{3 a}\right)
$$

Comparison with the numerical integration of eq 5.5 shows that for $\chi=0$ the difference is only a few percent, whereas for $\chi=0.5$ eq 5.9 may overestimate the contact potential by some $10 \%$. For the example of Figure 7 eq 5.4 gives $-W_{\mathrm{s}}(0)=1.911,1.908,1.923$, and 1.849 for $\chi$ $=0,0.2,0.4$, and 0.5 , respectively, whereas eq 5.9 predicts $1.918,1.950,2.073$, and 2.064 for these four solvencies. In Figure 8 we have (data from eq 5.9 in parentheses) $(a=5) 0.466(0.473)$ for $\chi=0$ and 0.457 (0.475) for $\chi=0.4 ;(a=10) 0.947$ (0.967) for $\chi=0$ and $0.916(0.974)$ for $\chi=0.4 ;(a=20) 1.925$ (1.968) for $\chi=$ $0,1.850$ (2.001) for $\chi=0.4$, and 1.680 (1.903) for $\chi=$ 0.5 .

The most important conclusion is that $W_{\mathrm{s}}(0)$ is of order $a \varphi_{\mathrm{b}}$ in all cases, for any solvency. Equation 5.9 is a reasonable approximation for the contact potential.

5.3. Trends for the Pair Potential. We could try the same procedure of taking the integrand out of the integral for the pair potential $W_{\mathrm{s}}(h)$ in eq 5.4. This would lead to

$$
W_{\mathrm{s}}(h) \approx-\frac{4}{3} a \varphi_{\mathrm{b}} \frac{\left(\delta_{\mathrm{s}}-h / 2\right)^{2}}{\delta_{\mathrm{c}}^{2}}\left(1+\frac{2 \delta_{\mathrm{s}}}{3 a}+\frac{h}{6 a}\right)
$$

The even simpler Deryagin-like approximation of Louis et al. ${ }^{9}$ reads

$$
W_{\mathrm{s}}(h)=-\frac{4}{3} a \varphi_{\mathrm{b}} \frac{\left(\delta_{\mathrm{s}}-h / 2\right)^{2}}{\delta \delta_{\mathrm{s}}}
$$

and matches with eq 5.10 when the last factor in eq 5.10 is taken unity for any $a$ and $\delta_{\mathrm{c}}{ }^{2}$ is replaced by $\delta \delta_{\mathrm{s}}$. In this case the approximations are less satisfactory: eq 5.10 underestimates the range of attraction by roughly a factor 1.5 in semidilute solutions (and this underestimation is even stronger for eq 5.11). When $h$ is 
rescaled by this factor, eq 5.10 would describe the curves in Figures 7 and 8 quite reasonably (apart from the tail end of the interaction, where the full integration of eq 5.4 gives a stronger attraction). It is easy to understand why the range of attraction is wider than $2 \delta_{\mathrm{s}}$ : when using only $\delta_{\mathrm{s}}$, a range is selected which corresponds only to the final value $\varphi_{\mathrm{b}}$, whereas in the integration of eq 5.4 smaller concentrations with a higher range also contribute. For the planar case we corrected for this effect by introducing a precisely defined interaction distance $\delta_{\mathrm{i}}$ which is larger than $\delta$. For spherical geometry such a correction is more difficult because the $h$ dependence is more complicated, and the expression for the "spherical" depletion thickness $\delta_{\mathrm{s}}$ is more involved than that of the "planar" depletion thickness $\delta$.

We conclude this section with a remark on the simple "overlap Ansatz". For spherical geometry, eq 3.8 is modified to $W(h)=-\Pi_{\mathrm{b}} V_{\mathrm{ov}}$, with $V_{\mathrm{ov}}$ given by eq 5.3 . To compare this with eq 5.10 , we may replace $\Pi_{b}$ by $\Omega(\infty) / \delta_{\mathrm{i}}$ (eq 4.9), where $\delta_{\mathrm{i}}$ is the interaction distance for flat plates. With the approximation $\Omega(\infty)=(2 / 9) \varphi_{b} / \delta$ according to eq 4.1 we end up with a result for $W(h)$ which is similar to eq 5.10: the only difference is that the factor $1 / \delta_{\mathrm{c}}{ }^{2}$ is replaced by $1 /\left(\delta \delta_{\mathrm{i}}\right)$. The result is of the same order of magnitude but differs from eq 5.10 (which is already inaccurate as to the range) by (at least) a factor of 2 . This is because $\delta_{\mathrm{c}}$ is smaller than $\delta$, which in turn is smaller than $\delta_{\mathrm{i}}$. Therefore, for spherical geometry the underestimation of the "overlap Ansatz" is more serious than for flat plates, where the underestimation was a factor $\delta / \delta_{i}$; see the discussion at the end of section 4.3

To test these theories experimentally, one needs to directly measure the force between particles. This is nowadays possible using atomic force microscopy ${ }^{22}$ or total internal reflection microscopy for the pair potential between a flat wall and a sphere ${ }^{23,24}$ or optical tweezers for the potential between two spheres. ${ }^{25}$ An appropriate test for our simple explicit equations for the concentration and solvency effects would be experimental studies where the polymer-solvent quality is systematically varied. We are not aware of such a systematic study but hope this work could trigger new experiments.

\section{Some Remarks on the Consequences for Colloidal Stability}

Measurements of the osmotic pressure or scattering techniques allow the determination of the effective osmotic second virial coefficient, ${ }^{26,27}$ defined as

$$
B_{2}=2 \pi \int_{0}^{\infty} r^{2}\left(1-\mathrm{e}^{-W_{\mathrm{s}}(r)}\right) \mathrm{d} r
$$

where $r=h+2 a$ is the distance between the particle centers. This virial coefficient is often taken as a measure of the stability of a colloidal suspension at low colloid volume fractions. For the interaction between two hard spheres in a solution containing nonadsorbing polymer

$$
B_{2}=\frac{16}{3} \pi a^{3}+2 \pi \int_{0}^{\infty}(h+2 a)^{2}\left(1-\mathrm{e}^{-W_{\mathrm{s}}(h)}\right) \mathrm{d} h
$$

In the second term we changed the integration variable $r$ (lower limit $r=2 a$ ) to $h$ (lower limit $h=0$ ). Results for $\tilde{B}_{2} \equiv B_{2} /\left(4 \pi a^{3} / 3\right)$ as a function of polymer concentration are given in Figure 9, for $N=1000$, three colloid radii $(a=5,10$, and 20$)$, and for $\chi=0$ (dashed curves)

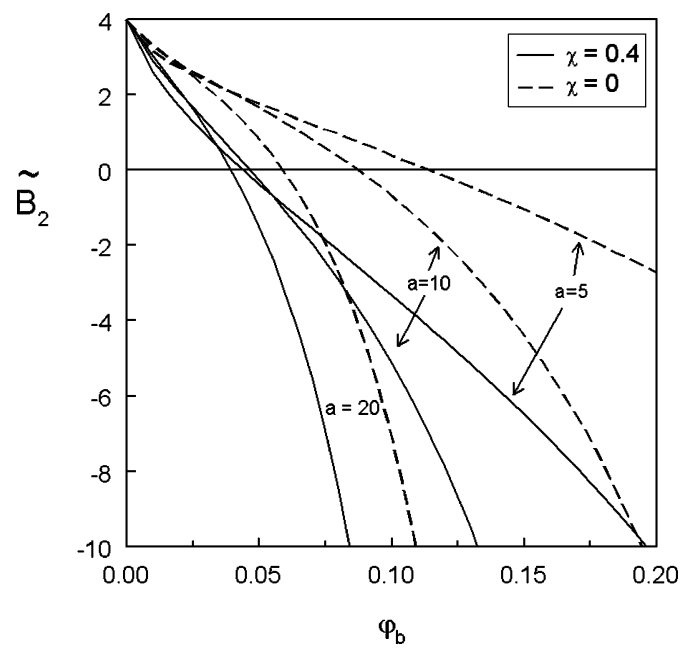

Figure 9. Osmotic second virial coefficient $\tilde{B}_{2} \equiv B_{2} /\left(4 \pi a^{3} / 3\right)$ for $a=5,10$, and 20 as a function of the polymer volume fraction $\varphi_{\mathrm{b}}$, for a chain length $N=10^{3}$. The solid curves correspond to $\chi=0.4$, and the dashed curves represent $\chi=0$. The curves were computed from eq 6.2, using eq 5.4 for $W_{\mathrm{s}}(h)$.

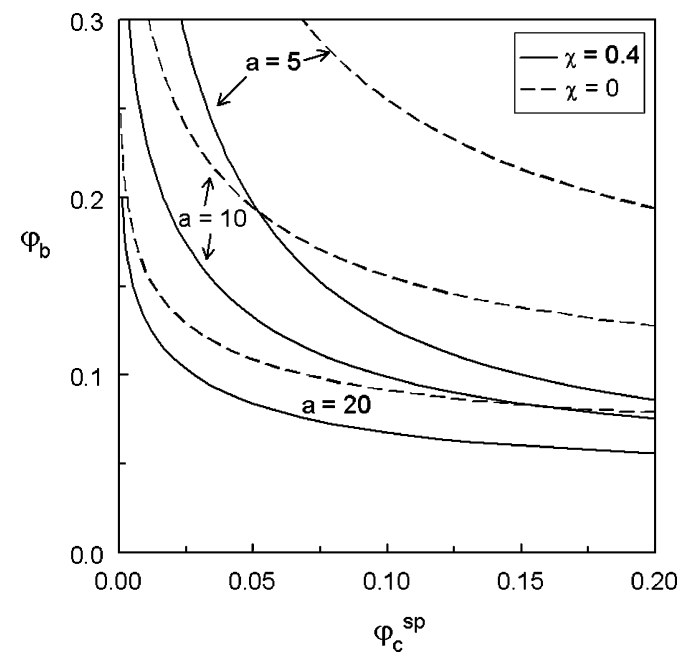

Figure 10. Spinodal curves $\varphi_{\mathrm{b}}\left(\varphi_{\mathrm{c}}^{\mathrm{sp}}\right)$ for $N=10^{3}, a=5,10$, and 20 , and $\chi=0$ (dashed curves) and $\chi=0.4$ (full curves). The spinodal concentration $\varphi_{\mathrm{c}}^{\mathrm{sp}}$ was computed from $\varphi_{\mathrm{c}}^{\mathrm{sp}}=$ $-1 / 2 \tilde{B}_{2}$, and the curves of Figure 9 were used to convert $B_{2}$ to the polymer concentration $\varphi_{\mathrm{b}}$.

and $\chi=0.4$ (solid curves). For $\varphi_{\mathrm{b}}=0$ there are only hard-core repulsive interactions $\left(\tilde{B}_{2}=4\right)$. As $\varphi_{\mathrm{b}}$ increases, the depletion interactions shifts $B_{2}$ in the negative direction. This decrease is slower for smaller particles (at fixed polymer size $N$ ). For small $a$ (e.g., $a$ $=5$ in Figure 10) $B_{2}$ even remains positive over a relatively wide range of concentrations (narrowing down for larger particles). This implies that colloidal dispersions are expected to be stable over a wide range of concentrations for $a<R$. For a better solvent (with a smaller depletion thickness) a wider miscibility region is expected on the basis of these simple $B_{2}$ calculations.

In a simple virial approximation the osmotic compressibility (with respect to colloid concentration) is, for the lowest order in colloid concentration, proportional to $1+2 \tilde{B}_{2} \varphi_{\mathrm{c}},{ }^{28}$ where $\varphi_{\mathrm{c}}$ is the colloid volume fraction. Since this compressibility is zero at the spinodal, ${ }^{28}$ the spinodal condition at low colloid volume fractions $\varphi_{\mathrm{c}}$ becomes

$$
0=1+2 \tilde{B}_{2} \varphi_{\mathrm{c}}^{\mathrm{sp}}
$$


Hence, $B_{2}$ yields a lowest-order estimate of $\varphi_{\mathrm{c}}^{\mathrm{sp}}$ at the spinodal. A spinodal curve $\varphi_{\mathrm{b}}\left(\varphi_{\mathrm{c}}^{\mathrm{sp}}\right)$ can thus be constructed from $\varphi_{\mathrm{c}}^{\mathrm{sp}}=-1 /\left(2 \tilde{B}_{2}\right)$, converting the (negative) $B_{2}$ values from Figure 9 to the polymer concentration $\varphi_{\mathrm{b}}$. Figure 10 gives such spinodal curves, again for $N=1000, a=5,10$, and 20, and $\chi=0.4$ (solid curves) and 0 (dashed curves). The spinodal curves shift to higher polymer concentrations for larger polymer-tocolloid size ratios and for better solvency. For even smaller radii than $a=5$ we would expect miscibility up to very high polymer concentrations. However, for $a / R<1$ three- and higher-body interactions dominate the phase behavior ${ }^{29}$ so an analysis of the fluid-fluid phase stability based upon only $B_{2}$ breaks down. Then an osmotic equilibrium theory forms a better alternative to describe the phase behavior, including the fluid-fluid and fluid-solid binodals. We will address these issues in a forthcoming publication.

\section{Conclusions}

For small polymer concentrations the influence of solvent quality within a mean-field treatment is negligible. For larger polymer concentrations, the effect is significant. For two plates we find an increase of the attraction at contact $\left(\sim \varphi_{\mathrm{b}} / \delta\right.$, where the depletion thickness $\delta$ decreases with increasing solvency) and a decreasing range in better solvents. This range is $2 \delta_{\mathrm{i}}$, and the interaction distance $\delta_{\mathrm{i}}$ depends in a similar way on solvency as $\delta$ (but in semidilute solutions is higher than $\delta$ ). For the interaction between two spheres, the contact potential is of order $a \varphi_{\mathrm{b}}$ and nearly independent of solvency. Qualitatively, this is because the contact potential is of order $\Pi_{b} V_{\text {ov }}$, where the bulk osmotic pressure $\Pi_{b}$ increases with solvency and the overlap volume $V_{\text {ov }}$ (roughly proportional to $\delta^{2}$ ) decreases; these opposing trends largely compensate each other. The interaction range for spheres is smaller than for plates for geometrical reasons but also here decreases with increasing solvency.

From the pair potential we computed the second virial coefficient and estimated the spinodal curves at low colloid concentrations. From this it follows that increasing the solvency leads to an increase in the colloidpolymer miscibility; here the decreasing depletion zone is the dominating effect. In a later publication we will investigate the full phase diagram of colloids plus polymer chains. In this case it is not enough to consider only pair interactions and higher-order effects have to be taken into account, e.g., by an osmotic equilibrium theory.

\section{References and Notes}

(1) Russel, W. B.; Saville, D. A.; Schowalter, W. R. Colloidal Dispersions; Cambridge University Press: New York, 1989.

(2) Asakura, S.; Oosawa, F. J. Chem. Phys. 1954, 22, 1255

(3) Asakura, S.; Oosawa, F. J. Polym. Sci. 1958, 33, 183.

(4) Vrij, A. Pure Appl. Chem. 1976, 48, 471.

(5) Eisenriegler, E. Phys. Rev. E 1997, 55, 3116.

(6) Eisenriegler, E. J. Phys.: Condens. Matter 2000, 12, A227.

(7) Tuinier, R.; Vliegenthart, G. A.; Lekkerkerker, H. N. W. J. Chem. Phys. 2000, 113, 10768.

(8) Bolhuis, P. G.; Louis, A. A.; Hansen, J. P.; Meijer, E. J. J. Chem. Phys. 2001, 114, 4296.

(9) Louis, A. A.; Bolhuis, P. G.; Meijer, E. J.; Hansen, J.-P. J. Chem. Phys. 2002, 117, 1893.

(10) Fuchs, M.; Schweizer, K. S. J. Phys.: Condens. Matter 2002, 14, R239.

(11) Tuinier, R.; Lekkerkerker, H. N. W.; Aarts, D. G. A. L. Phys. Rev. E 2002, 65, R060801.

(12) Tuinier, R.; Aarts, D. G. A. L.; Wensink, H. H.; Lekkerkerker, H. N. W. Phys. Chem. Chem. Phys. 2003, 5, 3707.

(13) Scheutjens, J. M. H. M.; Fleer, G. J. Adv. Colloid Interface Sci. 1982, 16, 361.

(14) Fleer, G. J.; Skvortsov, A. M.; Tuinier, R. Macromolecules 2003, 36, 7857.

(15) Tuinier, R.; Fleer, G. J. Macromolecules 2004, 37, 8754.

(16) Casassa, E. F. J. Polym. Sci., Part B 1967, 5, 773; Macromolecules 1969, 2,14 .

(17) Scheutjens, J. M. H. M.; Fleer, G. J. J. Phys. Chem. 1979 83, 1619. See: Fleer, G. J.; Cohen Stuart, M. A.; Scheutjens, J. M. H. M.; Cosgrove, T.; Vincent, B. Polymers at Interfaces; Chapman \& Hall: London, 1993; Chapter 4.

(18) Joanny, J. F.; Leibler, L.; de Gennes, P. G. J. Polym. Sci., Polym. Phys. Ed. 1979, 17, 1073.

(19) Fleer, G. J.; Skvortsov, A. M. Theory for concentration and solvency effects in size-exclusion chromatography of polymers. Macromolecules, to be submitted.

(20) Wijmans, C. M.; Leermakers, F. A. M.; Fleer, G. J. Langmuir 1994, $10,4514$.

(21) Deryagin, B. V. Kolloid Z. 1934, 69, 155

(22) Milling, A.; Biggs, S. J. Colloid Interface Sci. 1995, 170, 604

(23) Walz, J. Y. Curr. Opin. Colloid Interface Sci. 1997, 2, 600.

(24) Bechinger, C.; Rudhardt, D.; Leiderer, P.; Roth, R.; Dietrich, S. Phys. Rev. Lett. 1999, 83, 3960.

(25) Verma, R.; Crocker, J. C.; Lubensky, T. C.; Yodh, A. G. Macromolecules 2000, 33, 177.

(26) van Helden, A. K.; Vrij, A. J. Colloid Interface Sci. 1980, 78, 312 .

(27) Rouw, P. W.; Vrij, A.; de Kruif, C. G. Colloids Surf. 1988, 31, 299.

(28) De Hek, H.; Vrij, A. J. Colloid Interface Sci. 1981, 84, 409.

(29) Bolhuis, P. G.; Louis, A. A.; Meijer, E. J. Phys. Rev. Lett. 2003, 90, 068304 .

MA048571P 